\title{
„... in meinem eigensten Herzen bin ich geradezu Briefschwärmer". Bemerkungen zu Theodor Fontanes Briefwerk
}

"Je suis du fond du cour un fervent adepte de l'écriture de lettres". Remarques sur l'œuvre épistolaire de Fontane

“... in meinem eigensten Herzen bin ich geradezu Briefschwärmer”. Remarks on Fontane's Letters

Jana Kittelmann

\section{OpenEdition}

Journals

Édition électronique

URL : http://journals.openedition.org/ceg/606

DOI : $10.4000 /$ ceg.606

ISSN : 2605-8359

Éditeur

Presses Universitaires de Provence

Édition imprimée

Date de publication : 18 novembre 2016

Pagination : 89-105

ISBN : 979-10-320-0087-8

ISSN : 0751-4239

\section{Référence électronique}

Jana Kittelmann, « „... in meinem eigensten Herzen bin ich geradezu Briefschwärmer". Bemerkungen zu Theodor Fontanes Briefwerk », Cahiers d'Études Germaniques [Online], 71 | 2016, Online erschienen am: 18 Mai 2018, abgerufen am 25 November 2020. URL : http://journals.openedition.org/ceg/606 ; DOI : https://doi.org/10.4000/ceg.606 


\title{
„... in meinem eigensten Herzen bin ich geradezu Briefschwärmer." 1 \\ Bemerkungen zu Theodor Fontanes Briefwerk
}

\author{
Jana KITTELMANN \\ Martin-Luther-Universität Halle-Wittenberg
}

\section{Der , echte' Fontane}

Fontanes Briefen haftet etwas Verführerisches, Attraktives, ja gar „Zauberhaftes“ 2 an. Ganze Generationen von Lesern zogen sie in ihren Bann. ${ }^{3}$ Darunter war auch Marcel Reich-Ranicki, der in seinem Essay Fontane, der unsichere Kantonist dem „außerordentlich solide[n] Autor“ Fontane, der „als Romancier und Theaterkritiker, als Chronist, Biograph und Reiseschriftsteller“ stets fleißig und zuverlässig arbeitete, den Briefschreiber gegenüberstellt:

In der Korrespondenz suchte er den dringend benötigten Ausgleich: Andere spielten Domino, züchteten Rosen, hörten Musik oder verführten Mädchen. Er aber schrieb Briefe, Hunderte, Tausende. Hier gab er Stimmungen und Launen nach, redete unbekümmert auf den Adressaten ein, behauptete heute dies und morgen jenes, ließ sich zu raschen, bewußt überspitzen und bisweilen kaum diskutablen Äußerungen hinreißen, hier experimentierte er spielerisch mit Gedanken, Motiven und Formulierungen. Das Korrespondieren war sein Hobby, seine Passion. In den Briefen, nur in den Briefen erlaubte er sich ein „unsicherer Kantonist“" zu sein. ${ }^{4}$

Einer der Gründe der Begeisterung für die Briefe Fontanes, der im epistolaren Raum nicht immer als der charmante Plauderer, sondern häufig nörgelnd,

1. Fontane an Hanns Fechner, 3.5.1889, in Gotthard Erler (Hrsg.), Fontanes Briefe in zwei Bänden, Berlin, Weimar, Aufbau-Verlag, 1968, Bd. 2, S. 223.

2. Thomas Mann, Der alte Fontane, in Thomas Mann, Werke - Briefe - Tagebücher: Essays I, hrsg. von Heinrich Detering unter Mitarb. von Stephan Stachorski, Frankfurt a.M., Fischer, 2002, S. 245-274, hier S. 261.

3. Hans-Heinrich Reuter, der die Sammlung Von Dreißig bis Achtzig herausgab, kam zu dem Fazit: „Man darf den Satz wagen: Fontane würde zur großen deutschen Literatur gehören, auch wenn von ihm nichts überliefert wäre als seine Briefe.“ In Theodor Fontane. Von Dreißig bis Achtzig. Sein Leben in seinen Briefen, München, Nymphenburger Verlagshandlung, 1970, S. 8.

4. Marcel Reich-Ranicki, „Fontane, der unsichere Kantonist“, in ders., Nachprüfung. Aufsätze über deutsche Schriftsteller von gestern, Stuttgart, Deutsche Verlagsanstalt, 1980, S. 9-15, hier S. 12. 
verbittert und „hemmungslos“ 5 schimpfend daherkommt, dürfte der bestimmte Ton des „Briefschwärmer[s]“ sein, den man in jeder Zeile spürt. Fontane liebte das Briefeschreiben. Briefschreibetage waren für ihn Feier- und Erholungstage. So heißt es in einem Schreiben an Georg Friedländer:

\footnotetext{
Dies ist der dritte Wochentag und auch der dritte Briefschreibetag; ich erhole mich dabei, nachdem ich mich an meinem Roman (das mächtige alte Packet, das auch mal bei Ihnen lagerte) ganz dumm corrigirt habe. Hoffentlich zeigt sich in den Briefen die Nachwirkung davon nicht allzu sehr. ${ }^{6}$
}

Mit der Lektüre der Briefe verband sich von Beginn an das Gefühl, dass diese den ,echten' Fontane zeigen, wie es Ernst Bertram in einer frühen Studie zu Fontanes Briefen festhielt: „Dazu gibt selbst die Form dieser Briefe noch den ganz echtesten Fontane seiner Werke: wir besitzen in unserer ganzen Briefliteratur keinen persönlicheren Briefstil als den seinen. "7 Dem folgte Hans-Heinrich Reuter, der die Briefsammlung von Zwanzig bis Achtzig herausgab und formulierte: „scharf ausgeprägt sehen wir die Physiognomie des alten Fontane in seinen Briefen vor uns.“8 Für Fritz Mauthner, der in den Briefen „eine Seele“ entdeckte, „die wir so nicht gekannt hatten“ und die ihm wegen ihrer „kränkenden Lieblosigkeit“ „leise[n] Schmerz“ bereitete, war Fontane gar der „geniale Briefschreiber“ des gerade zu Ende gegangen 19. Jahrhunderts. ${ }^{9}$

$\mathrm{Zu}$ Authentizität und außerordentlicher Begabung Fontanes gesellten sich Fleiß und beste epistolare Vernetzung. Friedländer, der Schmiedeberger Amtsgerichtsrat, dem der alte Fontane beispiellos offene Briefe schrieb, ${ }^{10}$ war nur einer der über 400 Korrespondenzpartner, mit denen Fontane Briefwechsel pflegte. Darunter sind Namen wie Theodor Storm, Paul Heyse, Wilhelm Wolfsohn, Bernhard von Lepel, Ludwig Pietsch, Mathilde von Rohr und nicht zu vergessen die Ehefrau Emilie Fontane. Der Briefwechsel des Ehepaars Fontane, über mehr als fünf Jahrzehnte kontinuierlich geführt, bildet einen sowohl aus biographischer als auch werkgeschichtlicher Sicht bedeutenden Teil des Briefwerkes des Dichters, der mit seiner Familie ein „präzis terminiertes Korrespondenzprogramm“" absolvierte und eine Art „Familienbriefnetz“" ${ }^{12}$ aufbaute, wie ein Brief aus Thale zeigt:

5. Walter Hettche, „Nachwort“, in ders. (Hrsg.), Theodor Fontane. Briefe an Georg Friedländer, Frankfurt a.M., Insel, 1994, S. 445-461, hier S. 451.

6. Ibid., S. 326.

7. Ernst Bertram, „Theodor Fontanes Briefe“ (1910), in ders., Dichtung als Zeugnis. Frühe Bonner Studien zur Literatur, hrsg. von Ralph-Rainer Wuthenow, Bonn, Bouvier, 1967, S. 43-69, hier S. 49.

8. Reuter, Zwanzig bis Achtzig, S. 13.

9. Fritz Mauthner, „Theodor Fontane posthumus“, in Das literarische Echo 8, 1905, S. 157-161.

10. Hettche, Fontane an Friedländer, S. 452.

11. Helmuth Nürnberger, „Fontanes Briefstil“, in Hugo Aust (Hrsg.), Fontane aus heutiger Sicht, München, Nymphenburger Verlagshandlung, 1982, S. 56-80, hier S. 63.

12. Vgl. Regina Dieterle, Theodor Fontane und Martha Fontane. Ein Familienbriefnetz, Berlin et al., De Gruyter, 2002. 
Meine liebe Frau. Heute früh hatte ich Zeilen erwartet; ich find es aber andrerseits natürlich, wenn sie ausbleiben. Du hast jetzt eine ziemlich starke Correspondenz: George, Martha, ich, dazu wünsch ich Dir von Herzen, daß meine Abwesenheitswochen Dir Gelegenheit zu kl. Ausflügen etc. geben, die vielleicht unterbleiben, wenn ich da bin. Das Ordnen der alten Briefschaften ist außerdem anstrengend und zeitraubend. ${ }^{13}$

Die Korrespondenz mit Emilie erweist sich als beispielhaft für die vielen verschiedenen Lesarten, die Fontanes Briefe zulassen. Deren Reiz und Bedeutung liegen nicht zuletzt in der Gleichzeitigkeit von Familiärem und Literarischem, in dem Nebeneinander von Alltag und Künstlertum. So wird in einem 1879 verfassten Brief das Zubettgehen des Dichters mit einem Einblick in die Arbeit an der „Crayn-Novelle“14 (1882-83 als Schach von Wuthenow veröffentlicht) verbunden. Die 1863 von einer Sommerfrische auf Usedom geschriebenen Briefe, in denen Fontane einen Besuch in Swinemünde, der Stadt seiner Kindheit, wo er schon als 14jähriger "die junge Unbefriedigtheit des jungen Poetenherzens“ 15 empfunden hatte, schildert, erscheinen als Vorstudie zu dem 1893 erschienenen autobiographischen Roman Meine Kinderjahre. Im Falle seines Buches Kriegsgefangen bilden die Briefe an Emilie den Ausgangspunkt der später zunächst in der Vossischen Zeitung und schließlich in Buchform veröffentlichten Beschreibung seiner französischen Kriegsgefangenschaft. Dass der Theaterkritiker Fontane untrennbar mit dem Briefschreiber Fontane verbunden ist, zeigen die Briefe an die Tochter Martha, der wichtigen Korrespondenzpartnerin seiner späten Jahre. Hier ist unter anderem Fontanes Beschäftigung mit Gerhard Hauptmanns Drama Vor Sonnenaufgang dokumentiert:

Meine liebe Mete. Schon gestern Abend wollte ich Dir einen kl. Brief stiften, kam aber nicht dazu, weil ich anderweitig eine große Correspondenz hatte, darunter ein Brief an einen Herrn Gerhart Hauptmann, der ein fabelhaftes Stück geschrieben hat: „Vor Sonnenaufgang, soziales Drama, 5 Akte." Ich war ganz benommen davon. Mama natürlich wieder in Angst, ich ginge zu weit, ich engagirte mich ungebührlich; Durchgänger, Hitzkopf, „Jüngling“; nachdem nun aber gestern eine Karte von Brahm eingetroffen ist, der ganz meine Anschauung theilt, hat sie sich einigermaßen beruhigt. Ich allein kann nie Recht haben, es muß immer erst bestätigt werden, und wenn es durch Müller oder Schultze wäre. Dieser Hauptmann, ein wirklicher Hauptmann der schwarzen Realisten=Bande, welche letztre wirklich was von den Schillerschen Räubern hat und auch dafür angesehen wird, ist ein völlig entphraster Ibsen, mit anderen Worten ist das wirklich, was Ibsen blos will, aber nicht kann, weil er in seinen neben der realistischen Tendenz herlaufenden Nebentendenzen - die freilich in den letzten Stücken zur Haupttendenz geworden sind - mehr oder weniger verrückt ist und in zugespitzter Weise ins Phrasenhafte verfällt. ${ }^{16}$

13. Gotthart Erler (Hrsg.), Emilie und Theodor Fontane: Der Ehebriefwechsel, 3 Bde., Berlin, AufbauVerlag, 1998, hier Bd. 3, S. 253.

14. Ibid., S. 187.

15. Ibid., Bd. 2, S. 260.

16. Dieterle, Familienbriefnetz, S. 371 ff. Martha Fontane schenkte diesen Brief Gerhart Hauptmann zum 50. Geburtstag. 


\section{Editorische Desiderate}

Neben ihrer Relevanz als biographischer und zeitgeschichtlicher Quelle sind die Briefwechsel Fontanes zugleich Spiegel und Reflexionsfläche der Literatur- und Kulturgeschichte seiner Zeit. Die folgenden zwei kleinen Beispiele könnten dabei unterschiedlicher kaum sein: In der Jagiellonen-Bibliothek Krakau findet sich ein Schreiben des jungen Dichters an Karl August Varnhagen von Ense, zentrale Gestalt der Romantik und des Vormärz. In seinem Schreiben bittet Fontane um die Protektion von Alexander Jungs Buch Über Goethes Wanderjahre, das „noch immer als Manuskript umherirrt“. ${ }^{17}$ Fontanes Autograph versah der passionierte Handschriftensammler Varnhagen fein säuberlich mit dem Namen „Theodor Fontane“ und machte es so zum Objekt seiner berühmten Sammlung (Abbildung 1). Weniger respektvoll ging hingegen Fontane mit dem Brief eines jungen Mannes um, der dem „großen Meister“ für die wohlwollende Aufnahme seines Gedichtbandes „Larenopfer“ dankte. Fälschlicherweise hatte ihn Fontane wegen seines „zweiten Vornamen[s]“ für eine Frau gehalten: „ich bin männlichen Geschlechts und hoffe mich auch im Leben stets männlich im besten Sinn des Wortes, zu bethätigen“, schrieb etwas verschnupft Rainer Maria Rilke, dessen Brief in der Werkhandschrift von Von Zwanzig bis Dreißig überliefert ist. ${ }^{18}$ Die fließende Grenze zwischen literarischem Werk und privater Korrespondenz wird hier in der Materialität der Handschriften konkret greifbar. (Abbildung 2)

Rilkes, von dem Empfänger als offenbar nicht sonderlich wichtig eingestuftes Schreiben ist nur einer von tausenden Briefen, die Fontane hinterlassen hat. 5842 Briefe von ihm (nicht an ihn) sind in dem 1988 erschienenen Verzeichnis von Fontanes Briefen ${ }^{19}$ vermerkt. Die Dunkelziffer liegt deutlich höher. Ausdrücklich verwiesen die Herausgeber auf verlorene und verschollene Dokumente sowie Briefentwürfe Fontanes (z.B. in den bereits erwähnten Werkhandschriften), die bei der Erschließung keine Berücksichtigung finden konnten.

Das öffentliche und literaturgeschichtliche Interesse am Briefwerk Fontanes setzte bereits kurze Zeit nach dessen Tod mit Erscheinen der ersten Briefe im Jahr 1905 ein. ${ }^{20}$ Der von Fontanes Schwiegersohn Karl Emil Otto Fritsch

17. Fontane an Karl August Varnhagen von Ense, 10. Februar 1852, Sammlung Varnhagen, Bibliotéka Jagiellonska Kraków, V 59 . Die vollständige Transkription des Briefes ist abgedruckt in Christa Schulze, „Ein Briefwechsel zwischen Th. Fontane und K. A. Varnhagen von Ense aus dem Jahr 1852“, in Fontane Blätter 6/1, 1985, S. 3-5.

18. Auf die Briefentwürfe, insbesondere auf den Rilke-Brief, hat Wolfgang Rasch in seinem Beitrag „Rudimente des Briefverkehrs Fontanes in seinen Werkhandschriften. Beispiel und Befund“ auf der Tagung „Wie immer ihr Th. F. - Theodor Fontanes Briefe im Kontext“ am 18.9.2014 hingewiesen. Die Werkhandschrift Von Zwanzig bis Dreißig mit dem Rilke-Brief befındet sich in der Stiftung Stadtmuseum Berlin. Wolfgang Rasch danke ich auch für den Hinweis, dass dem vermutlich Anfang 1896 entstandenen Brief eine kleine Korrespondenz zwischen Rilke und Fontane vorausgegangen sein muss, die jedoch nicht erhalten geblieben ist.

19. Charlotte Jolles et al. (Hrsg.), Die Briefe Theodor Fontanes: Verzeichnis und Register, München, Hanser, 1988.

20. Vgl. zur Rezeptions- und Editionsgeschichte der Briefe: Helmuth Nürnberger, „'Der Mann der langen Briefe' und seine Leser: Die Hanser-Ausgabe“, in Hanna Delf von Wolzogen, 


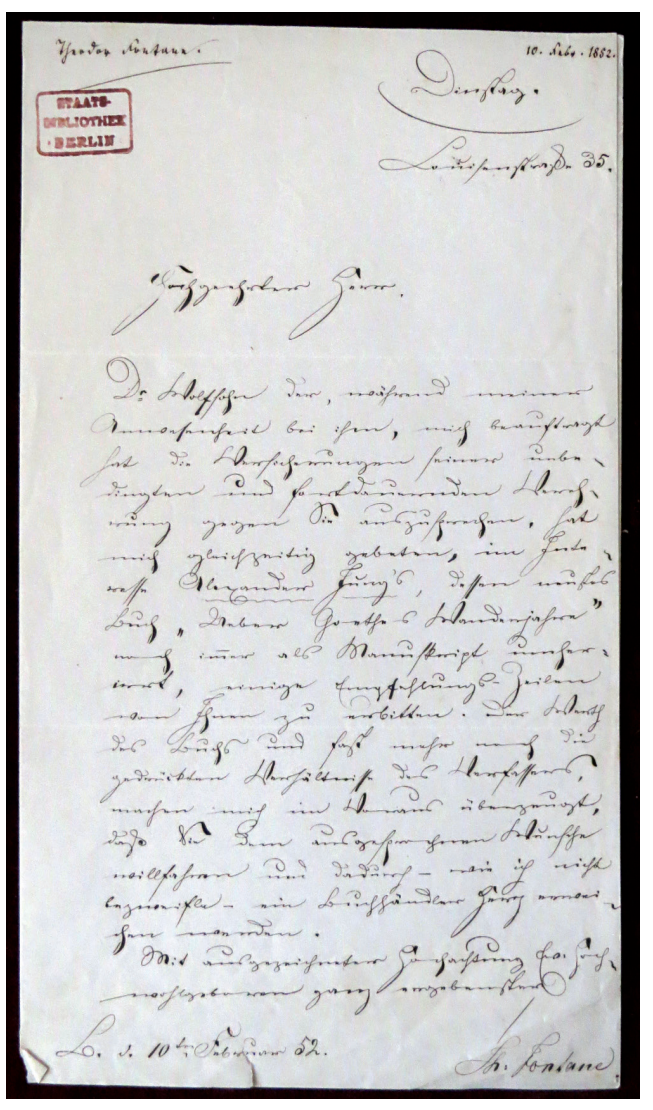

Abbildung 1 - Theodor Fontane an Karl August Varnhagen von Ense

Bibliotéka Jagiellonska Krákow, Sammlung Varnhagen, V 59.

Abbildung 2 - Rainer Maria Rilke an Theodor Fontane, Rückseite von Blatt 38 im Unterkonvolut von "Der achtzehnte März" (Hauptkonvolut "Von Zwanzig bis Dreißig"), Stiftung Stadtmuseum Berlin Foto: Wolfgang Rasch

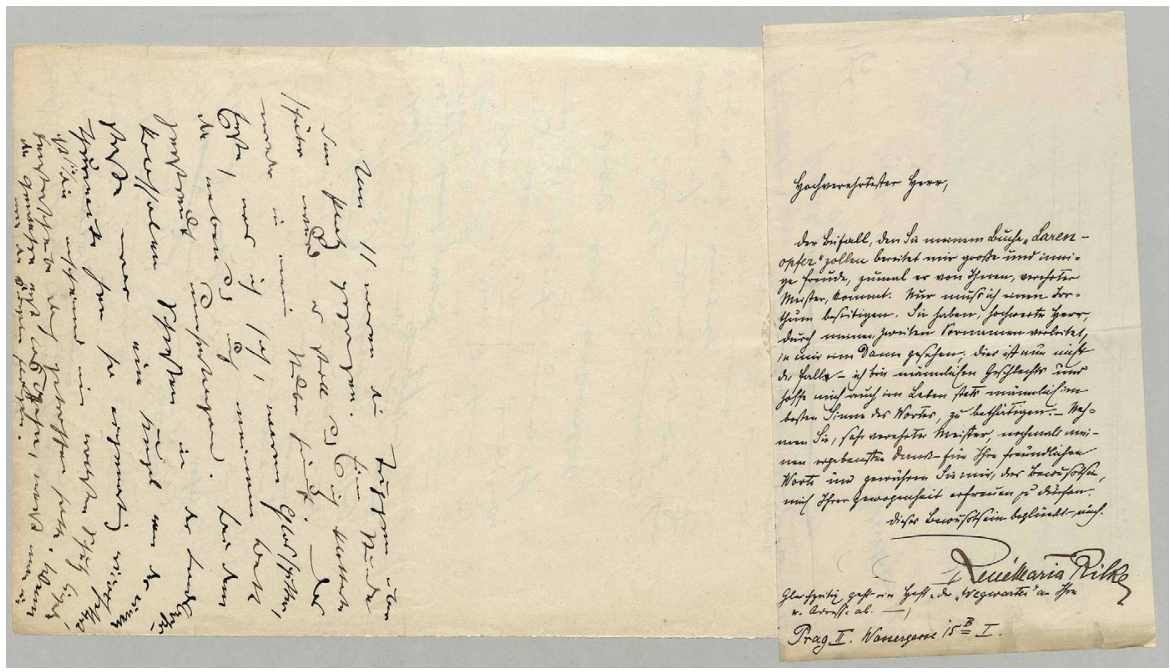


besorgten Ausgabe Theodor Fontane's Briefe an seine Familie folgten 1910 die von Paul Schlenther und Otto Pniower versammelten Briefe an die Freunde, die Thomas Mann in Begeisterung versetzen: „Sind noch mehr da? Man soll sie herausgeben!“21 schrieb er nach der Lektüre. Manns Forderung fand in einer bis heute anhaltenden Beschäftigung mit den Briefen ihr Echo. Zahlreiche Editionen und Neueditionen Fontanescher Korrespondenzen liegen vor. ${ }^{22}$ Eine Gesamtedition der in Archiven in ganz Europa und Nordamerika verstreuten Briefe Fontanes steht allerdings noch aus. Die „Erforschung der Briefe Fontanes und ihres Stils ist heute noch immer hauptsächlich ein Problem der Edition“, hatte Nürnberger bereits 1982 bemerkt und die Erschließung der Gesamtkorrespondenz als „wissenschaftliches Desiderat“ bezeichnet. $^{23}$

\section{Privat betreute Kunst}

Im Folgenden wollen wir uns nicht mit der Editionslage beschäftigen, sondern vielmehr einen Blick auf einige inhaltliche Aspekte von Fontanes Briefwerk, dessen „künstlerische Eigenständigkeit“ ${ }^{24}$ mittlerweile unbestritten ist, werfen. Dass es sich dabei nur um Ausschnitte und assoziative Bemerkungen zu einem großen Themenkomplex handeln kann, liegt auf der Hand. Am Beispiel ausgewählter Briefstellen soll insbesondere das enge Verhältnis zwischen dem Briefwerk und dem literarischen Werk des Dichters im Fokus stehen. Thomas Mann sprach in Noch einmal der alte Fontane von einer „künstlerisch betreut[en]“ 25 Privatheit, die kennzeichnend für Fontanes Korrespondenzen sei. Zugleich kann man im Umkehrschluss von einer privat betreuten künstlerischen Tätigkeit Fontanes sprechen, die in den Briefen dokumentiert ist. Denn trotz oder gerade weil die Briefe eine eigenständige künstlerische Rolle im Werk und der Biographie Fontanes einnehmen, sind die Grenzen zwischen den privaten Korrespondenzen und dem erzählerischen und journalistischen Werk des Dichters oft fließend. Was im privaten Raum des Briefes möglich ist und formuliert wird, geht bei Fontane nicht selten in die literarische Form, in seine Reisebeschreibungen, Theaterkritiken, Erzählungen und Romane über: „All meine geschichtliche Schreiberei, auch in den Kriegsbüchern, stützt sich am besten und wesentlichen

Rainer Falk (Hrsg.), Fontanes Briefe ediert, Würzburg, königshausen \&t neumann, 2014, S. 58-101.

21. Mann, Der alte Fontane, S. 245.

22. Der von Gabriele Radecke neu edierte Briefwechsel mit Theodor Storm, bei dem auch Briefbeilagen und nicht überlieferte, sogenannte erschlossene Briefe Berücksichtigung fanden, setzte dabei wegweisende editorische Standards.

23. Helmut Nürnberger, „Fontanes Briefstil“, S. 74. Das Theodor-Fontane-Archiv kündigte in einer Pressemeldung Ende 2014 eine elektronische Gesamtedition der Briefe an, schränkte jedoch ein, dass es „mehrere Jahre dauern“ werde, „ehe die Archivalien für das Forschungsportal aufbereitet seien.“ [http://www.heise.de/newsticker/meldung/Fontane-Dokumente-sollen-ins-Internetkommen-2507535.html], abgerufen am 23.11.2015.

24. Nürnberger, „Fontanes Briefstil“, S. 70.

25. Thomas Mann, „Noch einmal der alte Fontane“, in ders., Leiden und Größe der Meister. Gesammelte Werke in Einzelbänden, hrsg. von Peter de Mendelssohn, Frankfurt a.M., Fischer, 1982, S. 613-619, hier S. 615. 
immer auf die Briefe“ ${ }^{26}$ hatte Fontane an Hanns Fechner geschrieben. Die Briefe bestechen durch ein Oszillieren zwischen Literarizität und Privatheit. Sie sind angesiedelt zwischen „Bekenntnis und Prosaschule“ ${ }^{27}$, sie bieten Plaudereien und Stimmungen ebenso viel Platz wie Stilübungen und literarischer „Begleitmusik“28 zu den großen Romanen. Im Kontext des lebensweltlichen Gebrauches entstanden, erscheinen die Briefe dabei nicht selten als literarische Kunstwerke, ohne ihre Adressaten- und Alltagsbezogenheit zu verlieren.

\section{„ein bischen Plauderei"}

In einem Brief an die Stiftsdame und langjährige Freundin der Familie Fontane, Mathilde von Rohr, finden sich folgende Zeilen:

Mein gnädigstes Fräulein. Seit einer Anzahl von Wochen habe ich keinen Brief von unsrem hochverehrten Fräulein v. Rohr eintreffen sehn, woraus ich schließe, daß wir wohl an der Reihe sein werden. So dann ein bischen Plauderei. ${ }^{29}$

Was dann folgt, sind Nachrichten über den Alltag der Fontanes, die schwache Gesundheit der beiden Eheleute, von denen sich Emilie „im Ganzen gut gehalten“ hat, sehr zu Freude des Dichters, denn „eine verstimmte Frau ist ebenso ein Druck, wie eine heitre einem Flügel verleiht“. Die Entwicklung der Kinder, mit der es „erfreulich“ geht, ist ebenso Thema wie die Flucht vor der „täglichen Gesellschafts-Rennerei“. Allein die „Schopenhauer-Abende“ bei Wangenheims machten ihm noch Freude, heißt es, weil er „viel Anregung dadurch empfangen und verhältnismäßig wenig Zeit eingebüßt“ hat. Fontane erwähnt die Verlobung von Richard Lucae, der „lange herumgeliebt“ hat und nun mit seiner „kränklichen“ Braut nach Montreux gereist ist. Auch die Pflegetochter des Senators und Lyrikers Karl Eggers „hat sich mit einem jungen, blonden, dichtenden Ingenieur verlobt.“ Trocken fügt Fontane hinzu: „Das ganze Paar ist aber wenig dichterisch.“

Fontane plaudert, er ist ausführlich, ohne sich zu verlieren, unterhaltend, ohne lächerlich zu sein. Als „Mann der langen Briefe“ ${ }^{30}$ folgt er wie so oft auf mehreren Seiten einer epistolaren Choreographie, die seine Briefe zu einem Gespräch werden lassen und zudem wie ein inszeniertes Stimmungsbild wirken: „Ein Brief soll keine Abhandlung, sondern der Aus- und Abdruck einer Stimmung sein“31, hatte Fontane 1853 an Storm geschrieben. Trotz einzelner ästhetischer Überformungen und einer wohl durchdachten Konzeptionen der brieflichen Mitteilungen bleiben Fontanes Briefe jedoch immer im lebensweltlich-privaten Rahmen verhaftet. Sie sind alltägliche und zugleich zentrale Gebrauchsformen

26. Erler, Fontanes Briefe in zwei Bänden, Bd. 2, S. 223.

27. Nürnberger, „Fontanes Briefstil“, S. 62.

28. Mann, Noch einmal der alte Fontane, S. 614.

29. Otto Drude (Hrsg.), Fontane. Ein Leben in Briefen, Frankfurt a.M., Insel, 1981, S. 184.

30. Fontane in einem Brief an Karl Zöllner, 13.7.1881, in Theodor Fontane, Werke, Schriften und Briefe, Abt. IV/ Bd. 3, hrsg. von Helmut Nürnberger et al., München, Hanser, 1979, S. 157.

31. Gabriele Radecke (Hrsg.), Theodor Storm - Theodor Fontane. Briefwechsel, Berlin, Erich Schmidt, 2011, S. 38. 
seines Lebens. Fontane fiktionalisiert nicht, er kreiert sich keine ästhetischen Figurenkonstellationen oder geht gar einen ,epistolaren Pakt' mit seinen Adressaten ein, wie es für andere Briefwechsel der Zeit nachweisbar ist. ${ }^{32}$ In seinem Brief- und Gesprächsaustausch bleibt Fontane stets auf ein reales Gegenüber, einen konkreten Adressaten (oder Adressatin) fixiert und gleitet nie ins rein Monologische ab. So bemerkt auch Reich-Ranicki: „nicht das Monologische war seine Sache, sondern immer das Dialogische." ${ }^{33}$ In seiner spielerischen Übermittlung von mehr oder weniger wichtigen Nachrichten behält Fontane stets die für ihn typische Mischung aus Ironie und Distanziertheit, „Beobachtung und Plauderei, Anekdote und Reflexion“, ${ }^{34}$ aus Witz und charmantem Eingehen auf das Gegenüber bei. Das Plaudern wird zur Kunst erhoben. Der Brief avanciert zu dem Medium geselligen Verkehrs schlechthin. Fontane, der, wie die Äußerung an Mathilde von Rohr zeigt, der „Gesellschafts-Rennerei“ gern zu entfliehen suchte, konstituiert im einsamen Moment des Niederschreibens den Brief als virtuellen Geselligkeits- und Gesprächsraum, in dem er den wohl temperierten Ton $^{35}$ angibt.

Dieser Ton klingt weiter, wenn die Themen vom vermeintlich Alltäglichen ins Literarische hinübergleiten. Wie in so vielen anderen Briefen finden wir hier keinen abrupten, sondern einen geschickt vollführten Wechsel, der aus der vermeintlich alltäglichen Plauderei in ein reflexives Nachdenken über das eigene Schreiben mündet. Fontane berichtet von Änderungen im Wanderungen-Band Die Grafschaft Ruppin, um die Mathilde von Rohr gebeten hatte:

\begin{abstract}
Der Druck der 3. Auflage meiner Wanderungen in die dann auch das Rohr-Kapitel hineinkommen wird, schreitet rüstig vorwärts. [...] Ob die Familie [von Rohr, Anm. J.K.] im Ganzen dadurch befriedigt wird, muß ich bezweifeln, denn immer aufs Neue mache ich die Erfahrung, daß Familien, mit Ausnahme der gütigen, nachsichtigen und verehrten Dame, an die ich diese Zeilen richte, nicht zufrieden zu stellen sind. Ich glaube auch, daß sie, die Familien, von ihrem Standpunkte aus ganz Recht haben, weil ein Schriftsteller, der die Dinge lediglich als einen Stoff für seine Zwecke ansieht, auch bei größter Vorsicht und wirklichem Takt immer noch der Pietät entbehren wird, die im Herzen der Familienmitglieder lebt. Mitunter ist es freilich nicht mehr Pietät, sondern einfach eine Mischung von grenzenloser Dummheit mit ebenso grenzenloser Eitelkeit. ${ }^{36}$
\end{abstract}

Diese Zeilen nehmen viel von der Verbitterung des alten Fontane vorweg, der sich vor allem in den Briefen an Georg Friedländer skeptisch gegenüber seiner Umwelt zeigte und schonungslos mit dem preußischen Adel ins Gericht ging. Am 12. April 1894 fordert er in Abkehr von seinem „vielgeliebten Adel“: „Sie müssen alle geschmort werden. Alles antiquirt.“37

32. Vgl. zum Beispiel die Briefe Bettine von Arnims: Wolfgang Bunzel, „Ver-Öffentlichung des Privaten. Typen und Funktion epistolaren Schreibens bei Bettine von Arnim“, in Bernd Füllner (Hrsg.), Briefkultur im Vormärz, Bielefeld, Aisthesis, 2001, S. 41-96.

33. Reich-Ranicki, Der unsichere Kantonist, S. 11.

34. Nürnberger, Fontanes Briefstil, S. 65.

35. Vgl. zum Begriff „Fontane-Ton“ ibid., S. 57.

36. Drude, Fontane. Ein Leben in Briefen, S. 186.

37. Hettche, Fontane an Friedländer, S. 343. 
Doch schon die Briefe des frühen und mittleren Fontane zeigen, dass unter seiner Feder (meist eine Schwanenfeder) die harmloseste Plauderei und Alltagsschilderung schnell ins Spöttische, Bittere und Sarkastische umschlagen konnte. So schreibt er nach seinem Ausscheiden aus dem Diakonissenhaus Bethanien 1849 an Bernhard von Lepel:

\begin{abstract}
Mein lieber Lepel. Da sitz' ich wieder, und koste die Reize des Chambre garni. Die knarrende Bettstelle, die mitleidsvoll aus den Fugen geht, um einer obdachlosen Wanzenfamilie ein Unterkommen zu bieten - der wankelmüthige Nachttisch, - das geviertheilte Handtuch, die stereotypen Schildereien: Kaiser Nicolaus, und Christus am Kreuz; alles ist wieder da, mir Auge uns Herz zu erquicken. 0, es ist schön! ${ }^{38}$
\end{abstract}

Viele der Briefe dokumentieren die Schwierigkeiten eines von ständigen Geldsorgen geprägten Alltags. Sie sind Zeugnisse eines Lebens, in dem zunächst wenig klappen wollte. ${ }^{39}$ Verstimmt berichtet Fontane an Wilhelm von Merckel während eines Besuches in München, wo er sich auf Anregung Paul Heyses um die Stelle eines Privatbibliothekars des bayrischen Königs bewerben sollte:

Ich scheine unter keinem besondren Stern hier eingezogen zu sein. Wind und Regen von Anfang bis zu Ende, halbfuß tiefer Schmutz in den Straßen, Paul katharrlisch, Geibel zahnschmerzend und verschwollen, ich selber, geistig und körperlich, höchstens mit halber Dampfkraft arbeitend. ${ }^{40}$

Den nicht selten misslichen Umständen ihrer Entstehung zum Trotz versprühen Fontanes Briefe eine geradezu unerhörte „Mitteilungs- und Darstellungsfreude“ 41 , die von einer bestechenden Offenheit und Lauterkeit geprägt ist. Die Briefe an Mathilde von Rohr, Merckel und Lepel deuten an, dass Fontane in seinen Briefen nicht nur mit sich selbst, sondern ebenso mit vielen seiner Zeitgenossen, Kollegen und Gefährten wenig zimperlich umging. Lästereien, Meckereien, „Nörgeln und Nöhlen“42 standen auf der brieflichen Tagesordnung. Zu seinen ,Briefopfern' gehörten unter anderem Berthold Auerbach, Fanny Lewald und Karl Gutzkow. Am schlimmsten traf es vielleicht Theodor Storm. Den Besuch des berühmten Husumer Dichters, der scheinbar wenig Wert auf ein gepflegtes Äußeres legte, schlachtete Fontane genüsslich in einem Schreiben an den befreundeten Kunstschriftsteller Ludwig Pietsch aus:

Wenn ich Ihnen die Geschichte von Storm schon erzählt habe, so müssen Sie sie heut zum zweiten Mal hören. Als Rob. Prutz im sel. Deutschen Museum über ihn geschrieben hatte: „er sei sans phrase ein lyrischer Dichter, ein Dichter comme il faut" kam er in dem bekannten Kostüm mit langem hin und her baumelnden Wollshawl zu uns, erzählte von seinem Glück und wandte sich dann an meine damals noch hübsche Frau mit der Frage: ,Was rathen Sie mir, daß ich thue?' Ich war bei der ganzen Scene nicht zugegen und meine Frau schloß, als ich nach Hause kam, ihren Bericht darüber mit den Worten: ,ich hätt' ihm am liebsten

38. Radecke, Fontane-Lepel, Bd. 1, S. 162.

39. Fontane schreibt in einem Brief an Friedländer rückblickend einmal von seiner ,jämmerlichen Lebensgesammtstellung“. (Hettche, Fontane an Friedländer, S. 319)

40. Fontane, Briefe, IV/1, S. 657.

41. Nürnberg, „Fontanes Briefstil“, S. 62.

42. Vgl. Roland Berbig, „Vom Nörgeln und Nöhlen. Eine beiläufige Betrachtung zu Fontane und Kempowski“, in Fontane Blätter 95, 2013, S. 120-135. 
geantwortet, lassen Sie sich vor allem einen neuen Rock machen.' Wir beide können uns das Geständniß machen, daß er dessen in der Regel dringend bedürftig war. Brillante Lyriker waren nie Schneiderleute. ${ }^{43}$

Erfolgreiche Rivalen wie Storm gab Fontane unter dem Deckmantel der alltäglichen Plauderei nur allzu gern der Lächerlichkeit preis. Im intimen Raum des Privatbriefes fand dabei nicht selten Vorbereitung, was schließlich in den literarischen Werken der Öffentlichkeit übergeben wurde. Der brieflich verewigte Wollschal Storms fand Eingang in das „Storm“-Kapitel von Von Zwanzig bis Dreißig:

Storm war wie geschaffen für einen Tiergartenspaziergang an dichtbelaubten Stellen, aber für Kranzler war er nicht geschaffen. Ich seh' ihn noch deutlich vor mir. Er trug leinene Beinkleider und leinene Weste von jenem sonderbaren Stoff, der wie gelbe Seide glänzt und sehr leicht furchtbare Falten schlägt, darüber ein grünes Röckchen, Reisehut und einen Schal. Nun weiß ich sehr wohl, daß gerade ich vielleicht derjenige deutsche Schriftsteller bin, der in Sachen gestrickter Wolle zur höchsten Toleranz verpflichtet ist, denn ich trage selber dergleichen. Aber zu so viel Bescheidenheit ich auch verpflichtet sein mag, zwischen Schal und Schal ist doch immer noch ein Unterschied. Wer ein Mitleidender ist, weiß, daß im Leben eines solchen Produkts aus der Textilindustrie zwei Stadien zu beobachten sind: ein Jugendstadium, wo das Gewebe mehr in die Breite geht und noch Elastizität, ich möchte sagen, Leben hat, und ein Altersstadium, wo der Schal nur noch eine endlose Länge darstellt, ohne jede zurückschnellende Federkraft. So war der Stormsche. Storm trug ihn rund um den Hals herum, trotzdem hing er noch in zwei Strippen vorn herunter, in einer kurzen und einer ganz langen. An jeder befand sich eine Puschel, die hin und her pendelte. ${ }^{44}$

Andererseits war Fontane ein äußerst liebevoller Briefschreiber, der die Beobachtung von Alltäglichkeiten zum Beweis seiner Zuneigung für sein Gegenüber nutzte. Die Eigenart von Fontanes Briefen eröffnet sich nicht zuletzt in einer verstörenden Widersprüchlichkeit und einem bestechendem Nuancenreichtum. Ein auf der Reise nach England in Paris entstandener Brief an seinen „liebe[n] gute[n] Papa“ legt davon beredtes Zeugnis ab. ${ }^{45}$ Der Sohn schildert seinem Vater die Vorzüge der englischen gegenüber der französischen Metropole und sorgt sich zugleich wegen der Schwindelanfälle, die den alten Herrn plagen. Hier ist nichts von jenem epistolaren Zynismus und der „kränkenden Lieblosigkeit“ ${ }^{46}$ zu spüren, die man Fontane von verschiedenen Seiten vorwarf. ${ }^{47}$ Vielmehr sind die Zeilen durch und durch von der Zärtlichkeit des Sohnes für den Vater geprägt. Gleichwohl ist das Schreiben ebenso authentisch, wie der vorab zitierte Brief an Pietsch. Beide Male ist es Fontane, der hier spricht, unverstellt, wahr und authentisch. Seinem Credo, dass in einem

43. Fontane, Briefe, IV/2, S. 634.

44. Theodor Fontane, Von Zwanzig bis Dreißig, hrsg. von Gabriele Radecke und Heinrich Detering, bearb. von Wolfgang Rasch, Berlin, Aufbau-Verlag, 2014, S. 238.

45. Drude, Ein Leben in Briefen, S. 81-85.

46. Mauthner, Fontane Posthumus, S. 157.

47. Gotthard Erler verweist darauf, wie Fontane selbst den Vorwurf von seiner „Lieblosigkeit“ in einem Brief aus dem Jahr 1876 erörtert: „Egoistisch bin ich, aber nicht lieblos. Das ist ein großer Unterschied.“ Erler, Ehebriefwechsel, Bd. 1, S. XVII. 
Brief „der Schreiber leibhaftig vor Augen“48 treten müsse, ist er in seiner ganzen Widersprüchlichkeit selbst wohl am diszipliniertesten gefolgt.

\section{Die Briefe als „Stylübung[en]“}

$\mathrm{Zu}$ Reiz, Charme und Besonderheit des Fontaneschen Briefwerks gehört, dass die Briefe von Beginn an über ihre Mitteilungs- und Gesprächsfunktion hinaus häufig den Charakter von „Stylübung[en]“ besitzen. Dazu heißt es in einem Schreiben Fontanes an seinen Freund Lepel:

\section{Mein lieber Lepel}

Schon wieder mal! Fast muß es aussehn als gedächt' ich, in geschickter Benutzung errungner Portofreiheit, drauf los zu schmieren; - jeder Brief ist ja immer eine Stylübung und somit ein Schritt auf dem Weg - zur Vollendung. ${ }^{49}$

Fontane schrieb selbst dann gern und (wie wir gerade gehört haben) oft Briefe, wenn es gar nichts zu übermitteln gab. „Wir correspondiren jetzt á la Goethe und Bettina. Du hast häufıg nur Empfangsscheine für mich, und auch die nicht immer" 50 bemerkt er in einem weiteren Brief an Lepel, dessen Schreibfaulheit er immer wieder beklagte. Tatsächlich war Fontanes Briefschreibepensum selbst für das 19. Jahrhundert ungewöhnlich hoch. Nicht selten schrieb er allein um des Schreibens willen. Die Briefe waren Rückzugsort und boten zugleich einen Raum, in dem er sich als Schriftsteller ausprobieren und lebensnahe literarische Gebrauchsformen nutzen konnte, ohne sich einer breiten Öffentlichkeit stellen zu müssen.

Fontane, der sich gegenüber Zeitgenossen gern als „Mann der brieflichen Conversation“ "51 stilisierte, hatte dabei nicht den Anspruch, seine privaten Briefe als literarische Kunstwerke für ein breites Publikum zu konzipieren oder gar $\mathrm{zu}$ veröffentlichen. Vielmehr erschuf er sich in den Briefen eine eigenständige, dialogisch ausgerichtete Welt, deren Grenzen zu seinem literarischen Schaffen jedoch immer durchlässig und fließend waren. Lange vor dem großen Romancier gab es den Briefschreiber Fontane. Das Briefeschreiben hat er in einem Brief an Friedrich Stephany als „einzige absolute Promptheit“ ${ }^{52}$ seines Lebens bezeichnet. Zugleich sind die Briefe untrennbar mit der literarischen Entwicklung Fontanes verbunden. Sie dokumentieren seine Anfänge als Lyriker und Reiseschriftsteller ebenso, wie die Arbeiten an den großen Romanen, als deren „Begleitmusik“ Thomas Mann die Briefe des alten Fontane bezeichnete. Doch die Briefe begleiten nicht nur. Vielmehr geben sie etwas über den Autor preis, das in dessen Veröffentlichungen kaum mehr zu spüren ist. Sie eröffnen Einblicke in

48. Radecke, Fontane-Lepel, Bd. 1, S. 11.

49. Ibid., S. 287.

50. Ibid., S. 159.

51. Fontane, Briefe, IV/ 2, 151.

52. Ibid., IV/3, S. 466. 
Entwicklungsstadien von Ideen, Gedanken und stilistischen Versuchen. Völlig zurecht hat Nürnberger die Briefe Fontanes dessen „Prosaschule“ 53 genannt.

Der Briefwechsel mit Bernhard von Lepel ist dafür eines der besten Beispiele. Radecke arbeitete in der Neuedition der Korrespondenz zwischen Lepel und Fontane insbesondere die „literarische Dimension“ heraus und verwies darauf, dass die Briefe von „einer ungewöhnlichen Intimität und Offenheit, wie sie der junge Fontane offenbar gegenüber keinem anderen seiner Freunde gezeigt hatte“ 54 , zeugen. Die Poesie, das zeigt der Briefwechsel, erwies sich dabei als wichtigstes Bindeglied ${ }^{55}$ dieser Freundschaft. Die den Briefen beigelegten „Handschriften der eigenen poetischen Texte“"56, die Radecke erschlossen hat, zeigen, wie eng die Anfänge des Dichters Fontane, der 1850 mit der Sammlung Männer und Helden. Acht Preußen=Lieder auf dem literarischen Parkett reüssierte, mit dessen Briefen verbunden sind. Lepel nahm an der Entstehung der Gedichtsammlung regen Anteil. Per Brief schaltete er sich in den Entstehungsprozess der einzelnen Gedichte ein. Zuvor gab ihm Fontane „charte blanche“. ${ }^{57}$ Lepel konnte kritisieren, korrigieren, ergänzen und streichen, wie er es für richtig hielt. Die Position des Kritikers, dessen Bühne noch nicht das Feuilleton, sondern der Brief war, nahmen beide, Fontane und Lepel, gern ein. In ihren Briefgesprächen unterhielten sie sich nicht nur über „poetische Ausdrucksformen“, sondern erprobten diese „vielmehr sprachlich selbst“. ${ }^{58}$ Die Briefe - so betont Radecke - „fungierten darüber hinaus auch als eine Möglichkeit, sich in das Gebiet der journalistischen Literaturkritik einzuarbeiten, der sowohl Fontane als auch Lepel später professionell als Mitarbeiter verschiedener Zeitungen nachgegangen sind.“ "59

Wie kein zweiter erhielt Lepel Einblick in die von der Suche nach einer eigenen schriftstellerischen Identität geprägten Arbeit Fontanes, der im September 1849 nicht nur an seinen Gedichten, sondern ebenfalls an dem Dramenfragment Karl Stuart arbeitete:

Im Lapidarstyl geht es, trotz kleiner Unterbrechungen, tapfer weiter; vielleicht in 8 Tagen schon, kann ich Dir endlich (dies schon u. endlich macht sich gut) einen ganzen Akt zu Füßen legen. Die Übrigen, so denk ich, werden alle kürzer werden, einige wesentlich. Die Freude, die ich jetzt bei der Arbeit habe, kann ich Dir gar nicht beschreiben. Ich fühle mich jetzt wie ein Schachspieler, der mit seinem Gegner lange in hartem, heißem Kampf lag; jetzt mit einemmal übersieht er das ganze Spiel: den Springer hier - den Läufer dorthin, matt; die Sache ist abgemacht, man schiebt nur noch, der Plan ist fertig, der Sieg gewiß. [...] Der Erfolg dann draußen in der Welt, wer möchte den bestimmen?! ${ }^{60}$

Auf die ersten Erfolge seiner Arbeit musste Fontane freilich noch einige Zeit warten und auch die Freundschaft mit Lepel ebbte im Laufe der Jahre immer

53. Nürnberger, Fontanes Briefstil, S. 62.

54. Radecke, Fontane-Lepel, Bd. 2, S. 867.

55. Ibid.

56. Ibid., S. 869.

57. Ibid., Bd. 1, S. 363.

58. Ibid., Bd. 2, S. 869.

59. Ibid.

60. Ibid., Bd. 1, S. 158. 
mehr ab. An die Stelle des Kritikers und Empfängers literarischer Reflexionen trat immer mehr Emilie (später auch die Tochter Martha), die nicht nur die Romanmanuskripte Fontanes sorgfältig abschrieb, sondern von Beginn an Fontane in allen alltäglichen sowie literarischen Unternehmungen begleitete. Bereits dem dritten überlieferten Brief der Brautleute ist das Gedicht Der alte Ziethen beigelegt. Ob Verse wie „Sie stritten nie alleine / Der Ziethen und der Fritz, / Der Donner war der Eine, / Der andre war der Blitz“, die junge Emilie beeindruckten, bleibt freilich ihr Geheimnis. Die Gegenantwort ist nicht überliefert. Fontane war sich sicher, dass es seiner Braut gefallen würde und bat: „Schreibe mir Dein Urtheil; ich bin neugierig, - vor allen Dingen schreibe aber überhaupt recht viel und recht mit dem Herzen. “61

Spätestens seit Fontanes zweitem Aufenthalt in England, wo er mit dem Auftrag eine Deutsch-Englische Korrespondenz einzurichten für verschiedene Zeitungen tätig war, ist die mediale Vermischung von Feuilleton und Briefform unübersehbar. ${ }^{62}$ Im August 1856 notierte er für Emilie über eine Reise in Shakespeares Geburtsstadt, die auch das Briefpapier ziert:

\begin{abstract}
Meine liebe Frau. Nur wenige Worte auf diesem apart zu respektirenden Stück Papier, das dir das Häuschen zeigt worin Shakespeare zu Stratford am Avon geboren wurde. [...] Ich bin gestern um Mitternacht wieder hier eingetroffen. [...] Dir eine Beschreibung zu geben, ist unmöglich; das würde Tage kosten. Du wirst es entweder in der Vossin oder aber in meinem Tagebuche finden. ${ }^{63}$
\end{abstract}

Die Nähe von privater Briefberichterstattung und literarischer Produktion tritt hier offen hervor: Emilie, der Fontane den brieflichen Bericht verweigert, wird auf die Veröffentlichung der Reiseerlebnisse in der Vossischen Zeitung verwiesen. Andererseits nutzte Fontane bevorzugt die Form des Briefes für seine Berichte aus London. 1860 erschien nach seiner endgültigen Rückkehr von der britischen Insel: Aus England. Studien und Briefe über Londoner Theater, Kunst und Presse. Bereits 1854 wurde der Band Ein Sommer in London veröffentlicht, der aus den zumeist für die Preußische (Adler-) Zeitung verfassten Londoner Briefen hervorging. Briefliche Betreuung fand, wie so viele andere Werke, auch dieser Band:

\footnotetext{
Mit dem Druck meiner „Londoner Briefe“ wird es nun hoffentlich rascher gehn. Bitte, schreib mir immer was erschienen ist und auch $o b$ und wie es Euch gefallen hat. Die ersten Briefe glaub' ich sind die schwächeren und bin ich schon zufrieden wenn man ein bischen Esprit, Klarheit und stylistische Gewandheit an ihnen lobt. ${ }^{64}$
}

Allerdings fallen in der engen Verzahnung von Literatur und Brief häufig Ungleichheiten zwischen der poetischen Verarbeitung der Erlebnisse und den unmittelbaren Eindrücken in den Briefen auf. Diese Dissonanz illustrieren unter anderem Beispiele aus der Zeit von Fontanes französischer Kriegsgefangenschaft.

61. Erler, Ehebriefwechsel, Bd. 1, S. 5.

62. Vgl. dazu: Michael Ewert, „Uneigentliche Briefe. Zum Verhältnis von Briefen, Reisebriefen und Brief-Essays im Werk Fontanes“, in Fontanes Briefe ediert, S. 176-189.

63. Erler, Ehebriefwechsel, Bd. 1, S. 382.

64. Ibid., S. 101. 
Die während dieser Zeit entstandenen Briefe, die 1933 aus dem Familienbesitz versteigert worden sind und sich heute im Goethe-Schiller-Archiv Weimar befinden, zeigen, wie Fontane aus dem Briefeschreiben in die journalistische Arbeit übergeht. Am 10. November berichtet er Emilie von der Insel Oléron: „Meine Erlebnisse hab ich angefangen aufzuschreiben; mit der Hälfte bin ich ziemlich fertig. Sogar Verse habe ich gemacht. “65 Der Wechsel der Perspektive führt jedoch zu einer verschiedenen Darstellungsweise in Brief und Buch. Geschockt berichtete Fontane, dem zeitweilig die Hinrichtung als Kriegsspion droht, seiner Frau aus Landres am 6. Oktober 1870: „Meine Situation beschreibe ich dir nicht, der Hohn des Volkes ist furchtbar“66. In Kriegsgefangen hingegen lobt er den „humane[n] Zug“ der französischen Beamten, die französischen Mitgefangenen erscheinen als „liebenswürdig, gutherzig, neidlos“ ${ }^{67}$ Die Unmittelbarkeit des Briefes weicht hier der prosaischen Rückschau auf eine Reise, die aller Widrigkeiten zum Trotz als romantisches Abenteuer erzählt werden soll.

\section{Meister des brieflichen Gespräches}

Die Briefschwärmerei Fontanes griff auf dessen feuilletonistisches und erzählerisches Werk über. ${ }^{68}$ Gespräch und Brief erscheinen als zentrale Ausdrucksformen nicht nur seines privaten Alltags, sondern auch seines literarischen Werks. Die Belege dafür sind zahlreich und vielfältig. Sie reichen vom bloßen Zitat über die inhaltliche Wiedergabe bis hin zur Montage historischer und fingierter Briefe. In den Wanderungen etwa spielen die Briefe Dritter als kulturgeschichtliche Quelle eine bedeutende Rolle. Beispielsweise rekonstruiert Fontane einen Großteil seiner Schilderungen über Alexander von der Marwitz im Band Oderland aus dessen Briefwechsel mit Rahel Levin Varnhagen. Der Verfasser der Wanderungen fungiert dabei sogar als Editor, wenn er hier erstmals

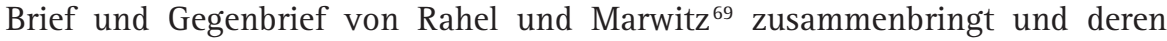
freundschaftlichen Briefwechsel als Dialog erscheinen lässt.

Zeigen uns die Wanderungen Fontane in erster Linie als Leser zeitgenössischer Briefsammlungen so lassen sich hingegen seine fingierten Briefe der Romane und Erzählungen durchaus als Teil seines Briefwerks verstehen. Die Verschmelzung von einer lebenslang erprobten und perfektionierten privatbrieflichen Kommunikation mit literarischen Darstellungsformen tritt in

65. Ibid., Bd. 2, S. 540.

66. Ibid., S. 521.

67. Theodor Fontane, Kriegsgefangen, in Sämtliche Werke: Aufsätze, Kritiken, Erinnerungen, Bd. 4: Autobiographisches, hrsg. von Walter Keitel, München, Hanser, 1973, S. 541-691, hier S. 583.

68. Vgl. zu den Romanbriefen Fontanes das Kapitel „Briefe, Selbstentwurf und Selbstkodifizierung“, in Claudia Liebrand, Das ich und die andern. Fontanes Figuren und ihre Selbstbilder, Freiburg, Rombach, 1990, S. 128-179.

69. Rahels Briefe waren in Rahel. Ein Buch des Andenkens (1834) und Marwitz' Briefe in Galerie von Bildnissen aus Rahel's Umgang und Briefwechsel (1836) einzeln von Karl August Varnhagen von Ense veröffentlicht worden. 
zahlreichen Beispielen des erzählerischen Werkes deutlich hervor, wenngleich man natürlich nie auf eine wortgetreue Wiedergabe persönlicher Mitteilungen in den Romanen stoßen wird. ${ }^{70}$ Gleichwohl gelangt Fontane auch als Romancier zu epistolarer Meisterschaft. Dabei tritt er als Erzähler weitaus zurückhaltender und versöhnlicher auf als in seinen Briefen. Dies zeigen beispielsweise seine Äußerungen über Bismarck. So heißt es über den Reichskanzler aus dem Munde Dubslavs, dieser sei ein „Zivil-Wallenstein“" ${ }^{71}$ In einem Brief an Martha Fontane findet sich hingegen folgende Beschreibung: „Er hat die größte Aehnlichkeit mit dem Schillerschen Wallenstein: Genie, Staatsretter und sentimentaler Hochverräther.“" ${ }^{72}$ Noch drastischere Worte findet Fontane ein Jahr später am „Bismarck-Tag mit wahrem Hohenzollernwetter“73:

\begin{abstract}
Diese Mischung aus Übermensch und Schlauberger, von Staatengründer und PferdestallSteuerverweigerer, [...] von Heros und Heulhuber, der nie ein Wässerchen getrübt hat erfüllt mich mit gemischten Gefühlen und läßt eine reine helle Bewunderung in mir nicht aufkommen. Etwas fehlt ihm und gerade das, was recht eigentlich die Größe leiht. Jude Neumann, uns gegenüber, hat auch nicht geflaggt, und Arm in Arm mit Neumann fordre ich mein Jahrhundert in die Schranken. ${ }^{74}$
\end{abstract}

Den von Thomas Mann so geschätzten „Altersradikalismus“ und die „ins Schwarze treffende Weltkritik“75 der späten Briefe hat man hier in Vollendung, in den Romanen sucht man solche Sentenzen freilich vergeblich.

Über die einzelnen Grenzverwischungen zwischen privatem und fiktivem Kommunikationsraum hinaus erweisen sich die lebensnahen Textformen Brief und Gespräch und damit der Dialog als Grundlagen aller Fontaneschen Romane. Dem Medium Brief kommt in den Romanen nicht selten eine Schlüsselrolle zu. Briefe sind Auslöser von Katastrophen (wie im Fall von Effi Briest), bestimmen den Fortgang der Geschichte und das weitere Schicksal der Protagonisten (wie etwa der Brief von Botho von Rienäckers Mutter in Irrungen, Wirrungen) oder sind selbst heimlicher Hauptakteur der Erzählung (wie etwa in Schach von Wuthenow).

Die Verschmelzung von privatbrieflicher Kommunikation, historischem epistolaren Material und fingierten Briefen lässt sich anhand der Novelle Schach von Wuthenow exemplarisch illustrieren. So spielt die Genese der Novelle unter anderem in der Privatkorrespondenz mit Martha Fontane eine wichtige Rolle. Martha nimmt regen Anteil an der Entstehung des Werks, wie ein Brief ihres Vaters vom 24. August 1882 zeigt, in dem er ihr für den „lieben Brief und die freundliche eingehende Beschäftigung mit dem armen Schach“76 dankt. Briefe

70. Vgl. dazu auch Nürnberger, „Fontanes Briefstil“, S 60.

71. Theodor Fontane, Der Stechlin, hrsg. von Klaus-Peter Möller, Berlin, Aufbau-Verlag, 2011, S. 364 .

72. Dieterle, Familienbriefnetz, S. 459.

73. Ibid., S. 465. Gemeint ist Bismarcks Geburtstag am 1. April.

74. Ibid.

75. Mann, Noch einmal der alte Fontane, S. 614.

76. Dieterle, Familienbriefnetz, S. 248. 
an Hermann Hauff sowie Hinweise in den Notizbüchern ${ }^{77}$ zeigen, dass Fontane Briefsammlungen wie Rahel. Ein Buch des Andenkens bei der Recherche verwendete, um vollends in dem von ihm geschaffenen Milieu des Briefes aufzugehen. Im Schach spielen bekanntlich Salonkultur und Briefnetzwerke der Romantik eine Hauptrolle. Zahlreiche historische Figuren treten auf und erhalten in Form von Briefen das Wort. In das Briefleben seiner Hauptfigur Victoire von Carayon, die angelehnt ist an die historische und für ihre Hässlichkeit berühmte Victoire von Crayen ${ }^{78}$, lässt Fontane all sein talent épistolaire einfließen. Victoire und ihre mehrseitigen Briefen an ihre (historisch nicht verbürgte) Freundin Lisette von Perbandt reiht sich mit der Stimme Fontanes in die Riege der großen Briefschreiberinnen um 1800 ein. Wie selbstverständlich wird der Brief zu ihrer zentralen Ausdrucksform der Selbstdarstellung und Selbsterkenntnis und darüber hinaus zu einem literarischen Kunstwerk. Einflüsse von Rahel Varnhagens Briefen an Freundinnen wie Regina Frohberg, Pauline Wiesel oder Adelheid von Carolath (die beiden letzteren tauchen ebenfalls in Fontanes Novelle auf), sind hier zu spüren. Wie diese findet Victoire bei Fontane allein in ihren Briefen einen Raum, ein eigenes Zimmer ${ }^{79}$, in dem sie über ihre Gefühle, ihre Ängste, ihr Schicksal, ihre Hässlichkeit, die Schach, der sie verführt hat, bei Fontane schließlich in den Selbstmord treibt ${ }^{80}$, schreiben kann:

Auf dem Heimwege tauschte Schach und führte mich. Er sprach sehr anziehend, und in
einem Tone, der mir ebenso wohl tat, als er mich überraschte. Jedes Wort ist mir noch in
der Erinnerung geblieben und gibt mir zu denken. Aber was geschah? Als wir wieder am
Eingange des Dorfes waren, wurd er schweigsamer und wartete auf die Mama. Dann bot er
ihr den Arm, und so gingen sie durch das Dorf nach dem Gasthause zurück, wo die Wagen
hielten und viele Leute versammelt waren. Es gab mir einen Stich durchs Herz, denn ich
konnte mich des Gedankens nicht erwehren, daß es ihm peinlich gewesen sei, mit mir und
an meinem Arm unter den Gästen zu erscheinen. In seiner Eitelkeit, von der ich ihn nicht
freisprechen kann, ist es ihm unmöglich, sich über das Gerede der Leute hinwegzusetzen,
und ein spöttisches Lächeln verstimmt ihn auf eine Woche. So selbstbewußt er ist, so
schwach und abhängig ist er in diesem einen Punkte. Vor niemandem in der Welt, auch vor
der Mama nicht, würd ich ein solches Bekenntnis ablegen, aber Dir gegenüber mußt ich es. ${ }^{81}$

Fontane nimmt als Erzähler die Rolle der Briefschreiberin Victoire ein und gelangt hier zu weitaus größter Meisterschaft als die historische Figur selbst. Das zeigt eine der wenigen epistolaren Spuren der historischen Victoire. In der Sammlung Varnhagen ist eines ihrer Billets an Rahel Levin Varnhagen (Abbildung 3) erhalten geblieben:

Mit unendlicher Freude nehmen wir Ihre freundliche Einladung gnädigste Frau an, könte ich aussprechen wie mein Herz mein bißchen Verstand Ihre hohen Eigenschaften versteht und verwahrt; so hätte die Welt noch nie einen solchen Brief gelesen als ich Ihnen heute

77. Vgl. Staatsbibliothek zu Berlin, Nachlass Theodor Fontane, Notizbuch E 2, I. Hier wird auf „die Briefe der Rahel“ als Lektüre verwiesen.

78. Victoire (geb. 1786) war die Tochter der Salonniere Henriette von Crayen.

79. Vgl. Virginia Woolfs 1929 erschienenen Essay A room of one’s own (dt. Ein eigenes Zimmer).

80. Otto Friedrich Ludwig von Schack, Vorbild des literarischen „Schach von Wuthenow“, nahm sich wohl wegen Spielschulden das Leben.

81. Theodor Fontane, Schach von Wuthenow, hrsg. von Gotthard Erler, Christine Hehle, Berlin, Aufbau-Verlag, 1997, S. 49. 
schreiben würde. So darf ich aber nicht wagen meine Gedanken aufzusetzen, und kann mich nur mit dem Gefühl begnügen daß Sie auch ein [im Original gestrichen] wenig Nachsicht für mich haben. Sorgen Sie ja nicht für eine Begleitung zu diesem Abend: wir gehen stets allein ich hoffe das Loos wird nicht wieder den Profeßor Gans treffen, der glaube ich wäre lieber die Schwalbe des Tobias für uns, als uns sicher zu geleiten.

Gnädige Frau

Ihre ergebene Victoire

Fontanes Victoire erhält und nutzt dagegen genau diese Chance, die der historischen Victoire offenbar verwehrt blieb: Gedanken aufzusetzen und Briefe zu schreiben, wie sie die Welt noch nicht gelesen hat. Nicht zuletzt das macht sie zu einem der vielen literarisch-epistolaren Alter Egos des großen Briefschwärmers Theodor Fontane.

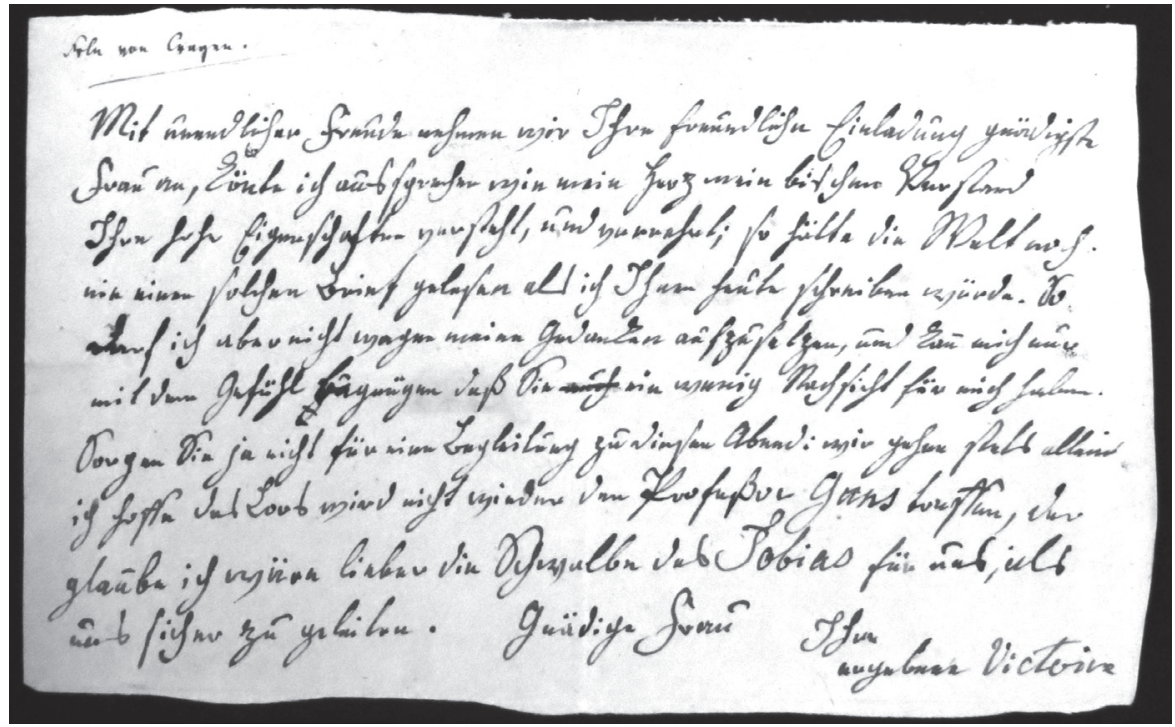

Abbildung 3 - Victoire von Crayen an Rahel Levin Varnhagen, ohne Ort (Berlin) und Datum Bibliotéka Jagiellonska Krákow, Sammlung Varnhagen, V 49. 